\title{
DIVERSITY OF FIRING PATTERNS IN A TWO-COMPARTMENT MODEL NEURON: USING INTERNAL TIME DELAY AS AN INDEPENDENT VARIABLE
}

\author{
Lei Wang*, Shenquan Liu ${ }^{\dagger}$ Yanjun Zeng
}

\begin{abstract}
Firing properties of single neurons in the nervous system have been recognized to be determined by their intrinsic ion channel dynamics and extrinsic synaptic inputs. Previous studies have suggested that dendritic structures exhibit significant roles in the modulation of somatic firing behavior in neurons. Following these studies, we show that finite information transmission delay between dendrite and soma can also influence the somatic firings in neurons. Our investigation is based on a two-compartment model which can approximately reproduce the firing activity of cortical pyramidal neurons. The obtained simulation results indicate that under subthreshold stimulus, spontaneous fast spiking activity is induced by large values of time delay, while for suprathreshold stimulus, regular bursting, chaotic firing and fast spiking can be observed under different time delays. More importantly, the transition mode between these diverse firing patterns with the variation of delay shows a period-doubling phenomenon under certain stimulus intensity. Consequently, our model results can not only illustrate the influential roles of internal time delay in the generation of a diversity of neuronal firing patterns, but also provide us with frameworks for investigating the impacts of internal time delay on the firing properties of many other neurons in the nervous system.
\end{abstract}

Key words: Compartment neuron model, internal time delay, firing patterns, ISI diagram

Received: October 16, 2012

Revised and accepted: May 13, 2013

\footnotetext{
${ }^{*}$ Lei Wang

Department of Mathematics, South China University of Technology, Guangzhou, 510640, China; Present address: School of Biomedical Engineering, Shanghai Jiao Tong University, Shanghai, 200240, China

${ }^{\dagger}$ Shenquan Liu

Department of Mathematics, South China University of Technology, Guangzhou, 510640, China

$\ddagger$ Yanjun Zeng

Biomedical Engineering Center, Beijing University of Technology, Beijing, 100022, China, E-mail: yjzeng@bjut.edu.cn
} 


\section{Introduction}

As the elementary units in the nervous system, neurons play key roles in the processing and transmission of neuronal signals, thus exploring what firing patterns neurons would produce under different conditions is of great importance in understanding how the brain functions. Previous investigations have reported that dendritic structure exhibits significant influences in modulating the firing behavior of neurons [1-5]. Based on two-compartment models, which consist of a soma and a dendrite, some researchers revealed that the smaller the soma is, the more irregularly the neuron would fire, and they also showed that two-compartment models can be used as slope detectors when the soma is small [6]. Computational study on models of pyramidal cells in the neocortex suggested that inter- and intraburst intervals in the neuronal spike trains can be affected by the total length of the apical dendrite and the topological structure of its branching pattern, also, these two factors can determine whether or not a neuron exhibits burst firing [7]. Recent experimental results on the layer $2 / 3$ pyramidal neurons indicated that hypercomplexity of the apical dendritic tuft could influence neuronal excitability by reducing the occurance of spike frequency adaptation [8]. In addition, there are still some researchers who have suggested that the experimentally observed firing patterns in neurons could be approximately reproduced using a two-compartment model by adjusting the electrical coupling strength between soma and dendrite [9].

Time delay is ubiquitous in most physical and biological systems, and it is inherent in the transmission of neuronal signals. As the information flow within neurons and between neurons is not generally instantaneous, taking into account the impacts of time delay in neurons and neuronal networks is thus reasonable.

During the past decades, many researches have revealed that finite information transmission delays could play significant roles in some neuronal systems. For instance, it has been suggested that time delays between neurons can facilitate and stabilize the synchronization of neural networks [10-13], induce multiple stochastic resonances under noisy environment [14-15], and modulate the oscillatory activity of neuron assemblies [16-17]. Moreover, in some neuronal circuits with the connection type of scale-free [18-19] and small-world [20-21], synchronous activity can switch from one state to another with the variation of time delay. Notably, in hybrid neuronal networks of the entorhinal cortex, shorter conduction delays tend to stabilize the synchronization of neuronal network with inhibitory couplings rather than excitatory couplings [22].

So far, researches related to the impacts of time delay in neuronal systems were mostly concentrated on coupled neurons and neuronal networks, while the impacts of information transmission delay within neurons are also very important, since neural signals transmit not only among neurons, but also within neurons, especially in neurons with long and complex dendritic structures. Moreover, the distributions of ion channels over a whole neuron membrane are not generally homogeneous, thus inevitably, the structure of neural signals would be affected by these distinctions when they transmit from one location to another in the neurons, this heterogeneities of ion channel distributions are also the reason for which a great deal of compartmental models for realistic neurons were developed [23-29]. 
Thus, in this study, the role of internal time delay on the firing patterns of single neuron is explored using a minimal two-compartment model neuron. Simulation results show that a diversity of firing patterns can be observed by varying time delay in a proper range, in addition, the transition route between different firing patterns with the variation of time delay is periodic under certain stimulus.

\section{Model and Methods}

The model neuron we employed to investigate is a two-compartment cortical pyramidal neuron, which is a simplified form the original Pinsky-Rinzel model [28]. This simplified model has been successfully used for capturing the complex bursting (an instance of square-wave bursting) found in the hippocampus of rats [30]. The schematic diagram of this minimal model is shown in Fig. 1, the active inward and outward ion currents which determine the compartment membrane potentials are also provided.

Mathematical descriptions for this two-compartment model neuron are modified from [30-31], as follows:

$$
\begin{gathered}
C_{m} \frac{d V_{s}}{d t}=-I_{\text {ions }}\left(V_{s}\right)+I_{d / s}+I_{s} \\
C_{m} \frac{d V_{d}}{d t}=-I_{\text {ions }}\left(V_{d}\right)+I_{s / d}+I_{d} .
\end{gathered}
$$

Here $I_{s}$ and $I_{d}$ are current inputs to the compartments. The corresponding ions in each compartment are:

$$
\begin{gathered}
I_{\text {ions }}\left(V_{s}\right)=g_{N a} m^{3} h\left(V_{s}-E_{N a}\right)+g_{K} n^{4}\left(V_{s}-E_{K}\right)+g_{\text {Leak }}\left(V_{s}-E_{\text {Leak }}\right) \\
I_{\text {ions }}\left(V_{d}\right)=g_{N a P} m_{\infty}^{3}\left(V_{d}-E_{N a}\right)+g_{K S} q\left(V_{d}-E_{K}\right)+g_{\text {Leak }}\left(V_{d}-E_{\text {Leak }}\right) .
\end{gathered}
$$

The coupling currents between the two compartments are:

$$
\begin{gathered}
I_{d / s}=\frac{g_{c}}{p}\left(V_{d}(t-\tau)-V_{s}\right) \\
I_{s / d}=\frac{g_{c}}{1-p}\left(V_{s}(t-\tau)-V_{d}\right) .
\end{gathered}
$$

In Eq. (5-6), $I_{d / s}$ denotes current from the dendritic compartment to the somatic compartment, conversely, $I_{s / d}$ denotes current from the somatic compartment to the dendritic compartment. $\tau$ is the term of internal time delay between the somatic and dendritic compartment.

The voltage-dependent gating variables are described using the standard HodgkinHuxley formalism.

$$
\begin{gathered}
\frac{d x}{d t}=\phi_{x} \frac{x_{\infty}(V)-x}{\tau_{x}(V)} \\
I_{y}=g_{y} a^{M} b^{N}\left(V-V_{y}\right)(y=N a, K, N a P, K S)
\end{gathered}
$$

In Eq. (7), $x$ is the activation or inactivation variable like $a$ and $b$ in Eq. (8). In Eq. (8), $g_{y}$ is the maximal channel conductance, and $V_{y}$ is the equilibrium potential. 
Other parameters and expressions contained in the model are as follows:

$$
\begin{aligned}
\alpha_{m} & =-0.1\left(V_{s}+31\right) /\left(\exp \left(-0.1\left(V_{s}+31\right)\right)-1\right), \quad \beta_{m}=4 \exp \left(-\left(V_{s}+56\right) / 18\right), \\
\alpha_{h} & =0.07 \exp \left(-\left(V_{s}+47\right) / 20\right), \quad \beta_{h}=1 /\left(\exp \left(-0.1\left(V_{s}+17\right)\right)+1\right), \\
\alpha_{n} & =-0.01\left(V_{s}+34\right) /\left(\exp \left(-0.1\left(V_{s}+34\right)\right)-1\right), \beta_{n}=0.125 \exp \left(-\left(V_{s}+44\right) / 80\right) \\
m_{\infty} & =1 /\left(1+\exp \left(-\left(V_{d}+57.7\right) / 7.7\right)\right), \quad q_{\infty}=1 /\left(1+\exp \left(-\left(V_{d}+35\right) / 6.5\right)\right), \\
\tau_{q} & =200 /\left(\exp \left(-\left(V_{d}+55\right) / 30\right)+\exp \left(\left(V_{d}+55\right) / 30\right)\right)
\end{aligned}
$$

The value of membrane capacitance $C_{m}$ is $1 \mu \mathrm{F} / \mathrm{cm}^{2}$. The ratio between the areas of the two compartments is described by the parameter $p=$ somatic area/total area whose value is 0.15 . Coupling conductance is $g_{c}=1 \mathrm{mS} / \mathrm{cm}^{2}$. The temperature scaling factors are $\varphi_{m}=10, \varphi_{h}=\varphi_{n}=3.33$. The ionic conductances are $g_{\text {Leak }}=0.18 g_{N a P}=0.12 g_{K S}=0.7 g_{N a}=55 g_{K}=20\left(\mathrm{mS} / \mathrm{cm}^{2}\right)$, and the equilibrium potentials are $E_{\text {Leak }}=-65 E_{N a}=55 E_{K}=-90(\mathrm{mV})$.

Simulations were performed using the MATLAB software, and the first-order Euler algorithm was employed to calculate the values of membrane potential with time step being $0.01 \mathrm{~ms}$. We only injected direct current into the dendritic compartment, and investigated the somatic action potential in this study. During our simulation, initial values of the somatic and dendritic membrane potential were set as $-64(\mathrm{mV})$, while initial values of the other gating variables were set as 0 . In order to avoid the influence of initial values, we disposed of the values in 0-500 (ms), and analyzed the somatic action potential values during 500-2000 (ms).

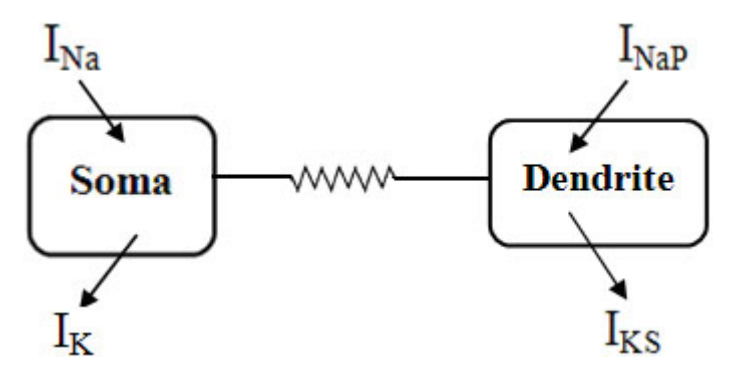

Fig. 1 Schematic diagram of two-compartment model for cortical pyramidal neuron.

\section{Results}

In analyzing the transitions between different firing patterns, the diagram of interspike intervals (ISI) is introduced, which has been widely used to describe the transition modes among various firing behaviors in neurons [32-35]. The value of ISI is represented as the time difference between adjacent peaks within spike trains, which is illustrated in Fig. 2.

To begin with, we provide examples of the somatic and dendritic membrane potential in the presence of internal time delay, and the results are demonstrated in Fig. 3, which vividly show that the somatic membrane potential and the dendritic 


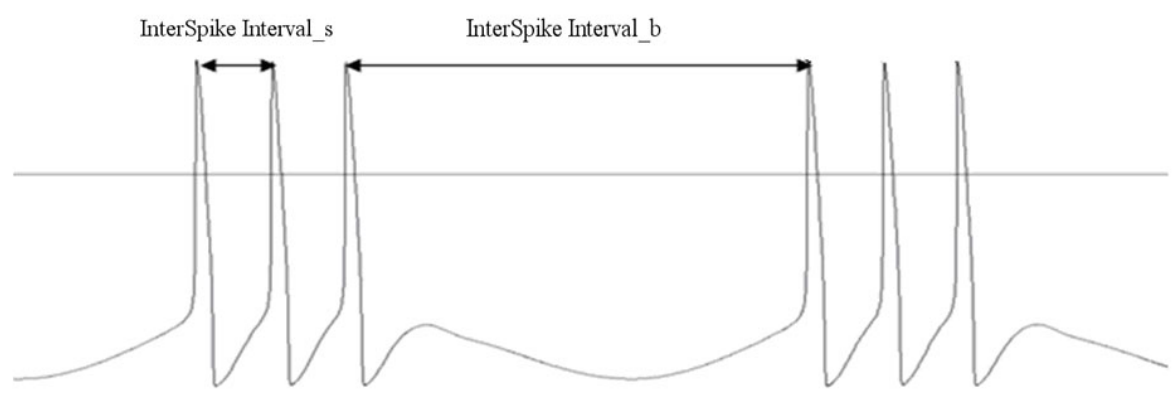

Fig. 2 The schematic of InterSpike Interval (ISI) in neuronal spike trains. InterSpike Interval_s denotes ISI between adjacent spikes within a burst, InterSpike Interval_b denotes ISI between adjacent bursts (modified from [36]).
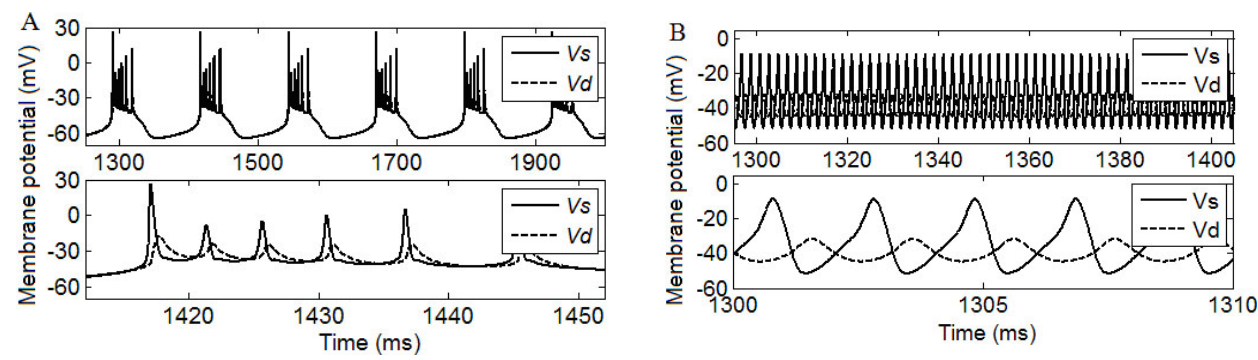

Fig. 3 Examples of the ping-pong phenomenon between the somatic and dendritic membrane potential in the presence of time delay when $I_{d}=3.0 \mu \mathrm{A} / \mathrm{cm}^{2}$. A: Regular bursting, B: Regular spiking. From $A$ to $B$, the value of delay is 0.2 and 0.53 respectively, and the bottom diagram is the enlargement of part of the top diagram in $A$ and $B$.

membrane potential vary interlaced, similar as the ping-pong phenomenon. In Fig. $3(\mathrm{~A})$, the initiation of membrane potential in soma would induce the generation of dendritic potential with relatively low amplitude, while during the intervals between interspikes, the amplitude of dendritic potential is higher than that of somatic potential, thus the generation of the next spike in soma may probably be caused by this higher-amplitude potential in dendrite. Similar case can be observed in Fig. 3(B), in which the value of delay is larger.

\subsection{Spontaneous activity induced by large values of time delay under subthreshold stimulus}

Traditionally, spontaneous firing activities of neurons are assumed to be evoked by their intrinsic ion channel dynamics [37-39]. For example, electrophysiological recordings on mouse retina demonstrated that spontaneous firing of retinal ganglion cells is induced by the activation of persistent sodium current and low threshold T-type calcium current [40]. In addition to these endogenous factors, experimental observation on the stratum oriens interneurons revealed that spontaneous cortical 
firings could be generated under extrinsic synaptic inputs [41]. Here we suggest that internal time delay within neurons can also play a potential role in the generation of neuronal spontaneous activities.
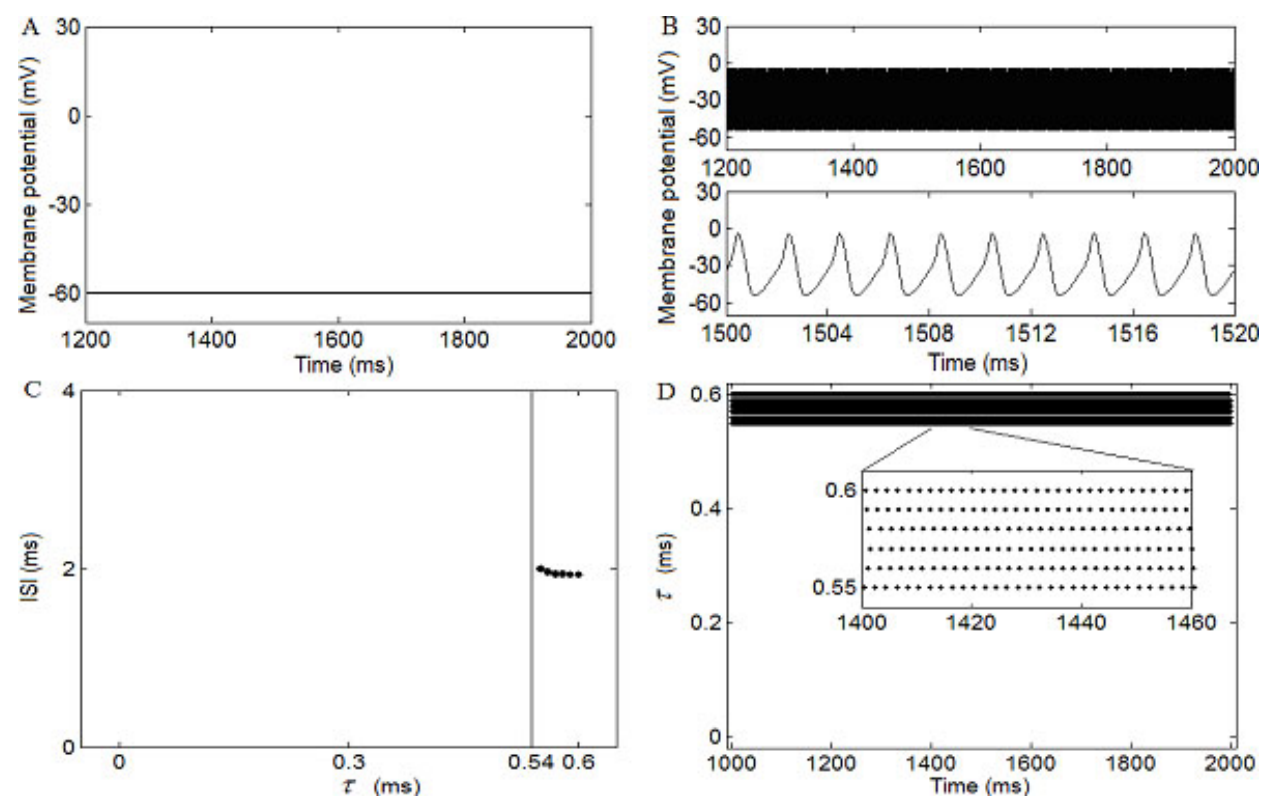

Fig. 4 Spontaneous fast spiking induced by internal time delay. Time courses of somatic membrane potential under different time delays, $A: \tau=0.54, B: \tau=$ $0.55 \mathrm{~ms}$. C: ISI diagram with respect to $\tau$ (the increment is 0.01), D: Raster plot of neuronal spikes under different delays. In $B$, the bottom diagram is the enlargement of part of the top diagram, and in D, the enlargement of part of the raster plot is provided.

Under subthreshold stimulus $\left(I_{d}=0.4 \mu \mathrm{A} / \mathrm{cm}^{2}\right)$, the neuron fires no spikes in the absence of $\tau$. While if $\tau$ is considered, small delays still failed to evoke any action potentials (Fig. 4(A)), thus the ISI values are zero. However, for larger time delays (bigger than 0.55), the fast spiking behavior is observed (Fig. 4(B)), and the ISI values are relatively small due to the dense spikes that the neuron fires. Simulated results in Fig. 4(C) and (D) could further manifest these spontaneous fast-spiking property evoked by internal time delay.

\subsection{Periodic bursting gradually switches to fast spiking with the increase of time delay}

Burst firing is prevalent in many biological and physical systems, especially in neural systems where it plays a pivotal role in neural information processing and transmission [42], and it has also been thought to have significant functions in reliable signaling [43-45] and synaptic plasticity [46-47]. The model neuron we investigated in this research is a simplified two-compartment model, which could 
successfully exhibit the complex bursting behavior of cortical pyramidal neurons. Thus in this section, we begin to study whether this complex bursting activity can be affected or regulated by neuronal internal time delays.
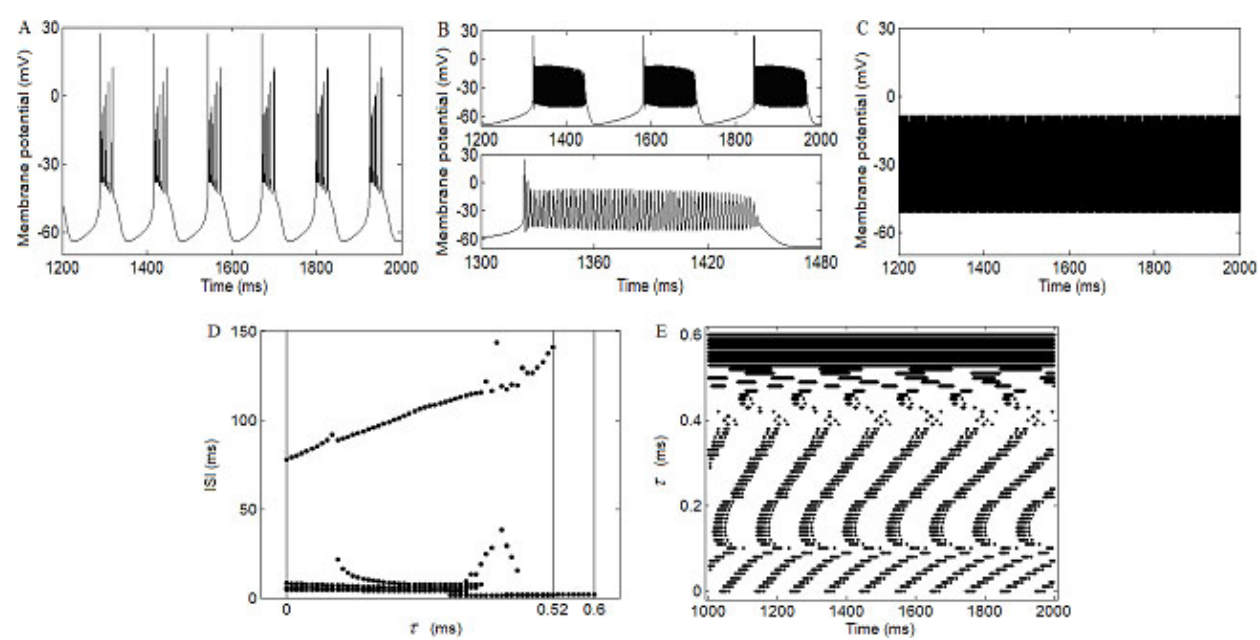

Fig. 5 Transition from periodic bursting to fast spiking with the increase of time delay. Time courses of somatic membrane potential under different time delays, $A: \tau=0.2, B: \tau=0.52, C: \tau=0.53 \mathrm{~ms}$, D: ISI diagram with respect to $\tau$ (the increment is 0.01). E: Raster plot of neuronal spikes under different delays. In B, the bottom diagram is the enlargement of part of the top diagram.

Simulation results in Fig. 5 vividly illustrate that the neuron can fire periodic bursting under a proper value of stimulus $\left(I_{d}=3.0 \mu \mathrm{A} / \mathrm{cm}^{2}\right)$ when internal time delay is not considered $(\tau=0)$. When we increase the delay, it is seen that the burst durations become longer and the spike number in each burst also increases with significance (Fig. 5(A-B)), however, when time delay reaches a critical value (0.53 in this simulation), the periodic bursting disappears but the fast spiking behavior emerges (Fig. 5(C)). ISI diagram and raster plot shown in Fig. 4(D\&E) further demonstrate the transition from periodic bursting to fast spiking induced by internal time delay.

\subsection{Chaotic firings intermittently occur with the increase of time delay}

In the absence of internal time delay, the model neuron fires chaotically when $I_{d}=4.0 \mu \mathrm{A} / \mathrm{cm}^{2}$, and small time delays maintain this irregular firing behavior, e.g. $\tau=0.03$ in Fig. 6(A). However, the spike patterns become much periodic when $\tau$ increases bigger than 0.03 but less than 0.15 , the corresponding instance is illustrated in Fig. 6(B) in which the value of $\tau$ is 0.1. The chaotic firings appear again when $\tau$ continues to increase from 0.16 to 0.19 , see Fig. $6(\mathrm{C})$ for more detail. A long duration of periodic burst firings then emerges when $\tau$ varies between 0.2 and 0.52 , the corresponding examples are shown in Fig. 6(D-E). Finally, the firing 

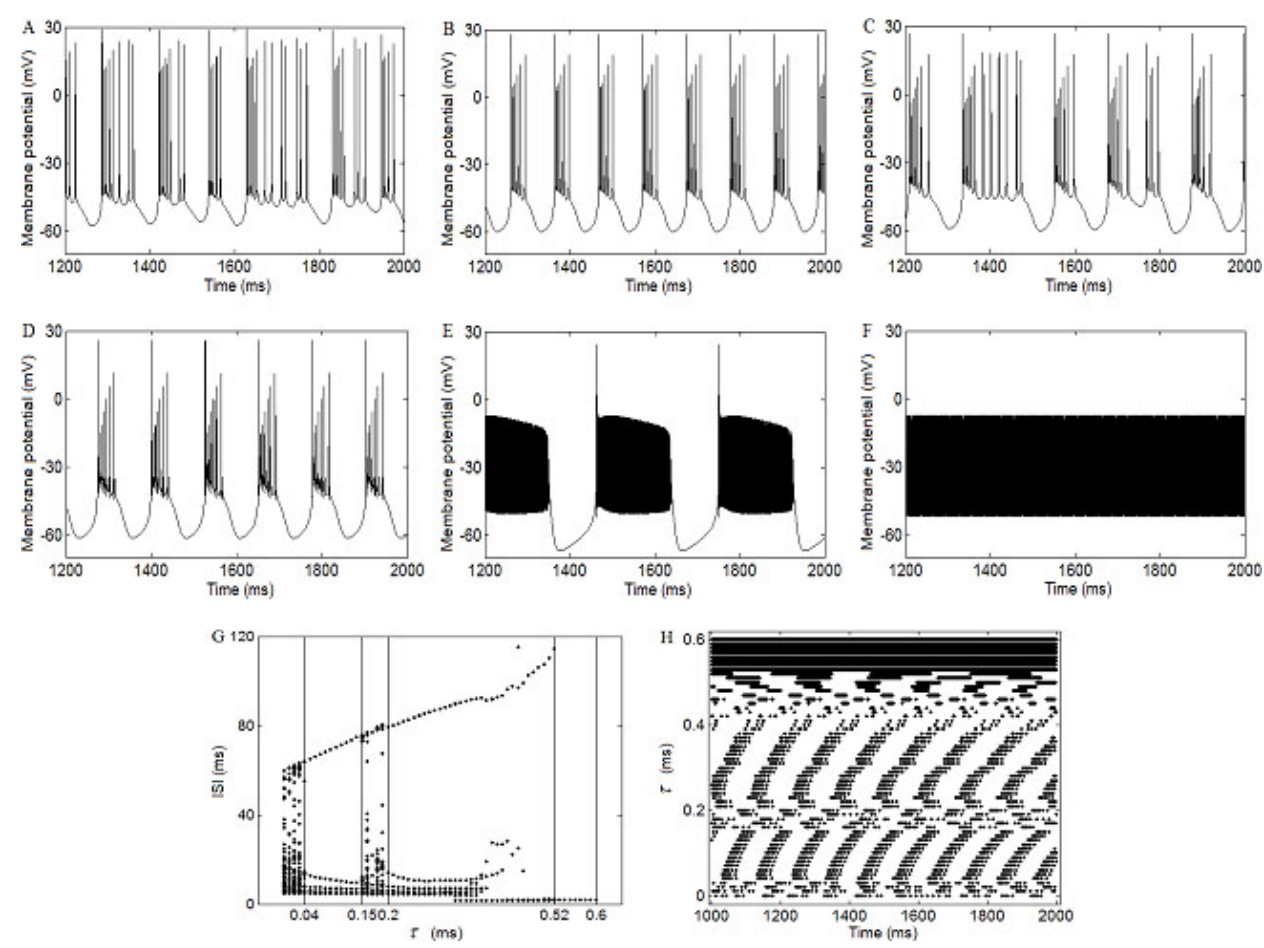

Fig. 6 Chaotic firings intermittently appear with the increase of time delay. Time courses of somatic membrane potential under different time delays, A: $\tau=0.03$, $B: \tau=0.1, C: \tau=0.19, D: \tau=0.3, E: \tau=0.52, F: \tau=0.53 \mathrm{~ms}, G:$ ISI diagram with respect to $\tau$ (the increment is 0.01), H: Raster plot of neuronal spikes under different delays.

pattern turns into fast spiking when $\tau$ is bigger than 0.52 (Fig. 6(F)). The ISI diagram and raster plot presented in Fig. $6(\mathrm{G})$ and $(\mathrm{H})$ can further describe how this complex transition mode is evolved.

\subsection{Period-doubling phenomenon induced by time delay under large stimulus}

In this section, we perform our simulations under a relatively larger stimulus, that is $I_{d}=4.7 \mu \mathrm{A} / \mathrm{cm}^{2}$, then we gradually increase the value of time delay the same as in Section 3.1-3.3. When $\tau=0$, the model neuron fires periodic spiking (Fig. 7(A)), while the spike patterns undergo a period-doubling cascade when $\tau$ increases from 0 to 0.32 , the corresponding transition route is clearly demonstrated in the insert figure of ISI diagram in Fig. 7(G). When we continue to increase the value of $\tau$, the irregular neuronal firings are induced (Fig. $7(\mathrm{C})$ ), and then, the neuron turns to fire periodic bursting with the increase of time delay (Fig. 7(D-E)), and finally, the fast spiking behavior appears with large value of $\tau$ (Fig. 7(F)). 

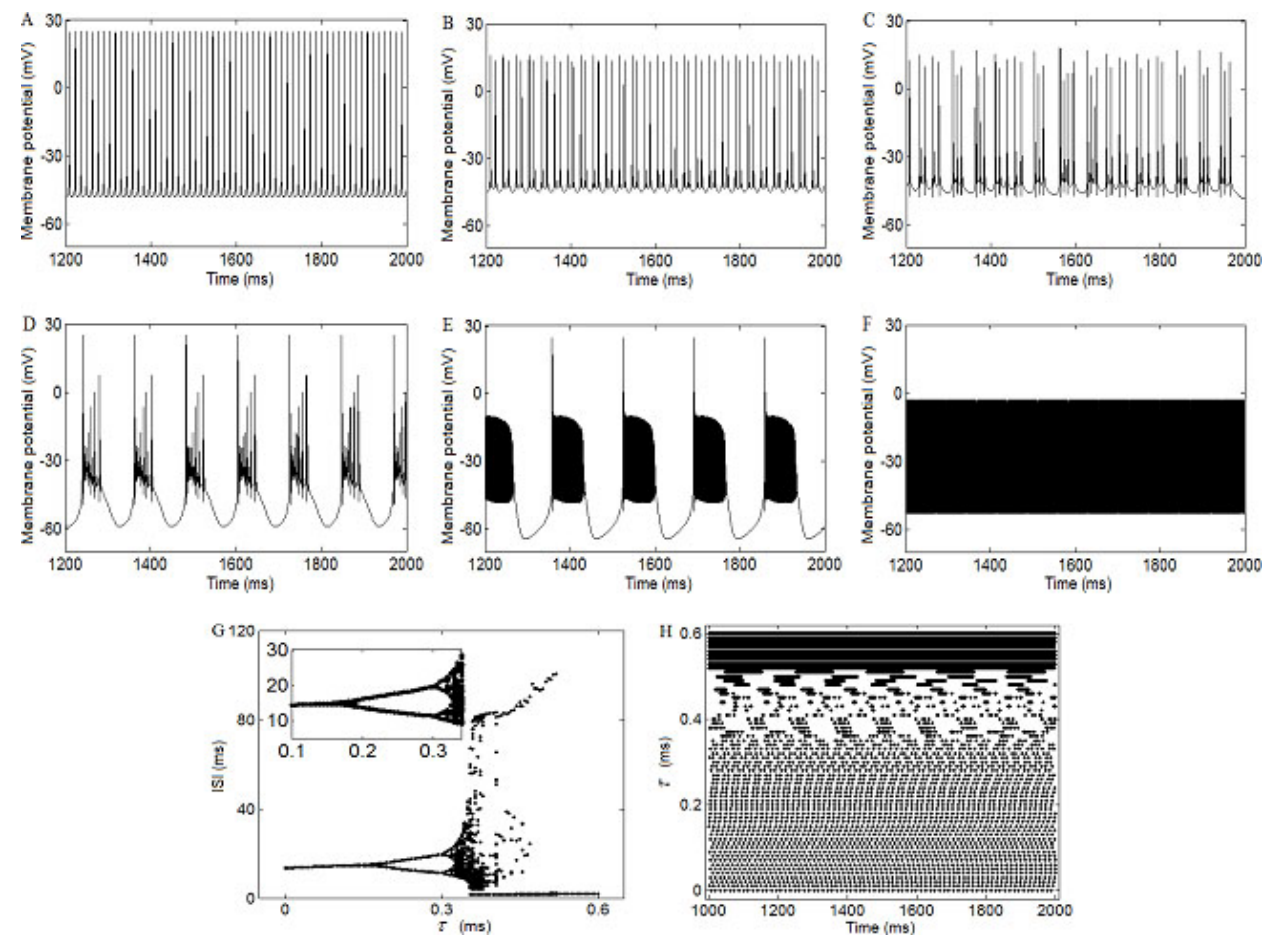

Fig. 7 Period-doubling phenomenon induced by internal time delay under large stimulus. Time courses of somatic membrane potential under different time delays, $A: \tau=0, B: \tau=0.28, C: \tau=0.35, D: \tau=0.39, E: \tau=0.5, F: \tau=0.55 m s, G:$ ISI diagram with respect to $\tau$ (the increment is 0.01), H: Raster plot of neuronal spikes under different delays.

\section{Discussions}

As the fundamental building elements in the nervous system, single neurons have been subject to extensive researches due to their complex firing properties and potential roles in information processing. Moreover, understanding how these exquisitely structured components behave under various environments is an important step in exploring how the brain works [48]. In the present study, we introduce an independent variable-internal time delay in a two-compartment model neuron, and investigate the firing patterns of this model neuron by changing the value of time delay. Simulation results indicate that a variety of firing patterns can be induced when the time delay varies in a proper range, and the transitions between these firing patterns appear to be periodic under some stimulus.

The influence of time delay on neuronal firing properties have been thoroughly investigated during the past decades. However, these studies were mainly concentrated on coupled neurons and neuronal networks. It is reasonable that information transmission from one neuron to the other neurons through the synapses are not generally instantaneous, which need delays to realize these processes. While 
we argue that neuronal signals transmitted within single neurons are also not instantaneous, especially when neurons have long and complex dendritic structures, accordingly the transmission of external synaptic inputs the dendrites receive to the soma needs time due to the complex morphology of neuronal dendrites. Our model results show that the existence of internal time delay between compartments displays functional effects in modulating the dynamical firing behavior of neurons.

In this study, the method we employed to describe the transition modes between different firing patterns is the ISI diagram, which has been widely used in the field of nonlinear analysis of neuronal systems [32-35]. Although widely applicable, and easy to be calculated numerically, ISI diagram has still failed to characterize the specific geometric structures of the corresponding bifurcation maps, thus for future considerations, other useful approaches, the phase plane analysis [59-50] and the fast/slow dynamical analysis [51-53] can be introduced to study the detailed bifurcation cascades like many other studies.

It should be noted that we mainly discussed the potential role of internal time delay on the firing patterns of single neurons in a two-compartment model, while real neurons in the nervous system often have complex morphologies, thus, subsequent attempts can concentrate on analyzing the influence of internal time delay in more complicated model neurons with multi-compartments. Moreover, due to the distinctions in the dynamical firing properties among different neurons, the numerical results about different firing pattern transitions shown in this paper may not be applicable, but the method we used here is universal and can be employed to study the firing properties of other neurons. Finally, internal time delay can also be introduced in neuronal networks or simply coupled neurons, and to investigate whether and how the variation of time delay within single neurons affects the whole activities of neuronal networks.

\section{References}

[1] Feng J., Li G.: Impact of geometrical structures on the output of neuronal models: a theoretical and numerical analysis. Neural Comput., 14, 3, 2002, pp. 621-640.

[2] Bastian J., Nguyenkim J.: Dendritic modulation of burst-like firing in sensory neurons. J. Neurophysiol., 85, 1, 2001, pp. 10-22.

[3] Duijnhouwer J., Remme M. W. H., van Ooyen A., van Pelt J.: Influence of dendritic topology on firing patterns in model neurons. Neucomputing, 2001, 38-40, pp. 183-189.

[4] van Ooyen A., Duijnhouwer J., Remme M. W., van Pelt J.: The effect of dendritic topology on firing patterns in model neurons. Network, 13, 3, 2002, pp. 311-325.

[5] Doiron B., Oswald A. M., Maler L.: Interval coding. II. Dendrite-dependent mechanisms. J. Neurophysiol., 97, 4, 2007, 2744-2757.

[6] Feng J., Li G.: Behaviour of two-compartment models. Neurocomputing, 38-40, 2001, pp. 205-211.

[7] van Elburg R. A., van Ooyen A.: Impact of dendritic size and dendritic topology on burst firing in pyramidal cells. PLoS Comput. Biol., 6, 5, 2010, e1000781.

[8] van der Velden L., van Hooft J. A., Chameau P.: Altered dendritic complexity affects firing properties of cortical layer $2 / 3$ pyramidal neurons in mice lacking the 5 -HT3A receptor. J. Neurophysiol., 108, 5, 2012, pp. 1521-1528.

[9] Mainen Z. F., Sejnowski T. J.: Influence of dendritic structure on firing pattern in model neocortical neurons. Nature, 382, 6589, 1996, pp. 363-366. 
Lei Wang, Shenquan Liu, Yanjun Zeng: Diversity of firing patterns...

[10] Ermentrout G. B., Kopell N.: Fine structure of neuronal spiking and synchronization in the presence of conduction delays. Proc. Natl. Acad. Sci., USA, 95, 3, 1998, pp. 1259-1264.

[11] Dhamala M., Jirsa V. K., Ding M.: Enhancement of neural synchrony by time delay. Phys. Rev. Lett., 92, 7, 2004, 074104.

[12] Rossoni E., Chen Y., Ding M., Feng J.: Stability of synchronous oscillations in a system of Hodgkin-Huxley neurons with delayed diffusive and pulsed coupling. Phys. Rev. E., 71, 2005, 061904 .

[13] Liang X., Tang M., Dhamala M., Liu Z.: Phase synchronization of inhibitory bursting neurons induced by distributed time delays in chemical coupling. Phys Rev E, 80, 2009, 066202.

[14] Wang Q., Perc M., Duan Z., Chen G.: Delay-induced multiple stochastic resonances on scale-free neuronal networks. Chaos, 19, 2, 2009, 023112.

[15] Yang X. L., Senthilkumar D. V., Sun Z. K., Kurths J.: Key role of time-delay and connection topology in shaping the dynamics of noisy genetic regulatory networks. Chaos, 21, 4, 2011, 047522 .

[16] Roxin A., Montbrió E.: How effective delays shape oscillatory dynamics in neuronal networks. Physica D, 240, 2011, pp. 323-345.

[17] Dorizzi B., Grammaticos B.: Delay-induced desynchronization in neuronal oscillations. Phys Rev A, 44, 10, 1991, pp. 6958-6961.

[18] Wang Q., Perc M., Duan Z., Chen G.: Synchronization transitions on scale-free neuronal networks due to finite information transmission delays. Phys Rev E, 80, 2009, 026206.

[19] Wang Q., Chen G., Perc M.: Synchronous bursts on scale-free neuronal networks with attractive and repulsive coupling. PLoS One, 6, 1, 2011, e15851.

[20] Tang J., Ma J., Yi M., Xia H., Yang X.: Delay and diversity-induced synchronization transitions in a small-world neuronal network. Phys. Rev. E., 83, 4, 2011, 046207.

[21] Hao Y. H., Gong Y. B., Lin X., Xie Y. H., Ma X. G.: Transition and enhancement of synchronization by time delays in stochastic Hodgkin-Huxley neuron networks. Neurocomputing, 73, $16-18,2010$, pp. 2998-3004.

[22] Wang S., Chandrasekaran L., Fernandez F. R., White J. A., Canavier C. C.: Short conduction delays cause inhibition rather than excitation to favor synchrony in hybrid neuronal networks of the entorhinal cortex. PLoS Comput. Biol., 8, 1, 2012, e1002306.

[23] Soto-Treviño C., Rabbah P., Marder E., Nadim F.: Computational model of electrically coupled, intrinsically distinct pacemaker neurons. J. Neurophysiol., 94, 1, 2005, pp. 590-604.

[24] Wang X. J.: Calcium coding and adaptive temporal computation in cortical pyramidal neurons. J. Neurophysiol., 79, 3, 1998, pp. 1549-1566.

[25] Crook S. M., Ermentrout G. B., Vanier M. C., Bower J. M.: The role of axonal delay in the synchronization of networks of coupled cortical oscillators. J. Comput. Neurosci., 4, 2, 1997, pp. 161-172.

[26] Booth V., Rinzel J., Kiehn O.: Compartmental model of vertebrate motoneurons for Ca2+dependent spiking and plateau potentials under pharmacological treatment. J. Neurophysiol., 78, 6, 1997, pp. 3371-3385.

[27] Li Y. X., Bertram R., Rinzel J.: Modeling N-methyl-D-aspartate-induced bursting in dopamine neurons. Neuroscience, 71, 2, 1996, pp. 397-410.

[28] Pinsky P. F., Rinzel J.: Intrinsic and network rhythmogenesis in a reduced Traub model for CA3 neurons. J. Comput. Neurosci., 1, 1-2, 1994, pp. 39-60.

[29] Zomorrodi R., Kröger H., Timofeev I.: Modeling thalamocortical cell: impact of ca channel distribution and cell geometry on firing pattern. Front. Comput. Neurosci., 2, 5, 2008.

[30] Kamondi A., Acsády L., Wang X. J., Buzsáki G.: Theta oscillations in somata and dendrites of hippocampal pyramidal cells in vivo: activity-dependent phase-precession of action potentials. Hippocampus, 8, 3, 1998, pp. 244-261.

[31] Kepecs A., Wang X. J.: Analysis of complex bursting in cortical pyramidal neuron models. Neurocomputing, 32-33, 2000, pp. 181-187. 
[32] Komendantov A. O., Kononenko N. I.: Deterministic chaos in mathematical model of pacemaker activity in bursting neurons of snail, Helix pomatia. J. Theor. Biol., 183, 2, 1996, pp. 219-230.

[33] Postnova S., Voigt K., Braun H. A.: Neural synchronization at tonic-to-bursting transitions. J. Biol. Phys., 33, 2, 2007, pp. 12-143.

[34] Postnova S., Finke C., Jin W., Schneider H., Braun H. A.: A computational study of the interdependencies between neuronal impulse pattern, noise effects and synchronization. J. Physiol., Paris, 104, 3-4, 2010, pp. 176-189.

[35] Wang L., Liu S., Zhang J., Zeng Y.: Burst firing transitions in two-compartment pyramidal neuron induced by the perturbation of membrane capacitance. Neurol. Sci., 33, 3, 2012, pp. 595-604.

[36] Wang L., Liu S. Q.: Neural circuit and its functional roles in cerebellar cortex. Neurosci. Bull., 27, 3, 2011, pp. 173-184.

[37] Choi Y. M., Kim S. H., Uhm D. Y., Park M. K.: Glutamate-mediated [Ca2+]c dynamics in spontaneously firing dopamine neurons of the rat substantia nigra pars compacta. J. Cell. Sci., 116, 13, 2003, pp. 2665-2675.

[38] Shuai J., Bikson M., Hahn P. J., Lian J., Durand D. M.: Ionic mechanisms underlying spontaneous CA1 neuronal firing in Ca2+-free solution. Biophys. J., 84, 3, 2003, pp. 20992111.

[39] Wang Y. C., Huang R. C.: Effects of sodium pump activity on spontaneous firing in neurons of the rat suprachiasmatic nucleus. J. Neurophysiol., 96, 1, 2006, pp. 109-118.

[40] Margolis D. J., Detwiler P. B.: Different mechanisms generate maintained activity in ON and OFF retinal ganglion cells. J. Neurosci., 27, 22, 2007, pp. 5994-6005.

[41] Yang E. J., Harris A. Z., Pettit D. L.: Synaptic kainite currents reset interneuron firing phase. J. Physiol., 578, 1, 2007, pp. 259-273.

[42] Izhikevich E. M., Desai N. S., Walcott E. C., Hoppensteadt F. C.: Bursts as a unit of neural information: selective communication via resonance. Trends Neurosci., 26, 3, 2003, pp. 161-167.

[43] Eyherabide H. G., Rokem A., Herz A. V., Samengo I.: Bursts generate a non-reducible spike-pattern code. Front Neurosci., 3, 1, 2009, pp. 8-14.

[44] Lisman J. E.: Bursts as a unit of neural information: making unreliable synapses reliable. Trends Neurosci., 20, 1, 1997, pp. 38-73.

[45] Oswald A. M., Doiron B., Maler L.: Interval coding. I. Bursting interspike intervals as indicators of stimulus intensity. J. Neurophysiol., 97, 4, 2007, pp. 2731-2743.

[46] Huerta P. T., Lisman J. E.: Bidirectional synaptic plasticity induced by a single burst during cholinergic theta oscillation in CA1 in vitro. Neuron, 15, 5, 1995, pp. 1053-1063.

[47] Wang X. J.: Fast burst firing and short-term synaptic plasticity: a model of neocortical chattering neurons. Neuroscience, 89, 2, 1999, pp. 347-362.

[48] Herz A. V., Gollisch T., Machens C. K., Jaeger D.: Modeling single-neuron dynamics and computations: a balance of detail and abstraction. Science, 314, 5796, 2006, pp. 80-85.

[49] Touboul J., Brette R.: Dynamics and bifurcations of the adaptive exponential integrate-andfire model. Biol. Cybern., 99, 4-5, 2008, pp. 319-334.

[50] Naud R., Marcille N., Clopath C., Gerstner W.: Firing patterns in the adaptive exponential integrate-and-fire model. Biol. Cybern., 99, 4-5, 2008, pp. 335-347.

[51] Tsaneva-Atanasova K., Osinga H. M., Riess T., Sherman A.: Full system bifurcation analysis of endocrine bursting models. J. Theor. Biol., 264, 4, 2010, pp. 1133-1146.

[52] Osinga H. M., Tsaneva-Atanasova K. T.: Dynamics of plateau bursting depending on the location of its equilibrium. J. Neuroendocrinol., 22, 12, 2010, pp. 1301-1314.

[53] Channell P., Fuwape I., Neiman A. B., Shilnikov A. L.: Variability of bursting patterns in a neuron model in the presence of noise. J. Comput. Neurosci., 27, 3, 2009, pp. 527-542. 